\title{
DNases prevent clots formed by neutrophil extracellular traps
}

Vessel occlusion by clots formed from platelets and fibrin is a well-characterized mechanism in haemostasis and thrombosis. According to new research published in Science, a noncanonical mechanism of vascular occlusion is the formation of a clot from DNA fibres released by neutrophils during inflammation in a phenomenon called neutrophil extracellular traps (NETs). The degradation of these NETs and, therefore, the prevention of vascular occlusion is mediated in vivo by two deoxyribonucleases (DNases).

Inflammation is an important defence mechanism against invading microbes, but can become pathological if unresolved. During acute inflammation, neutrophils are

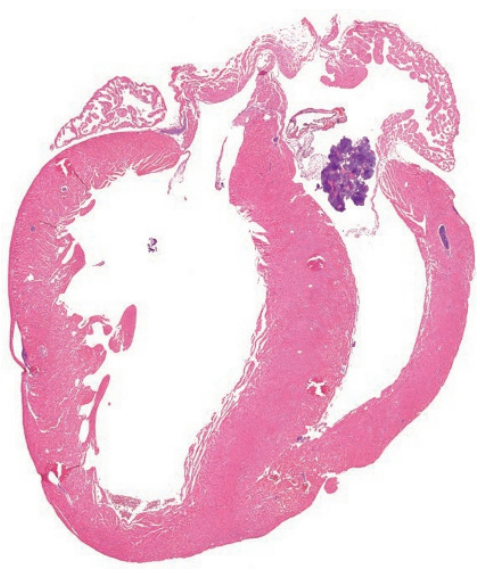

Large NET clot in the cardiac chamber and multiple smaller NET clots in the blood vessels. Image credit: Tobias A. Fuchs the predominant form of leukocyte. These neutrophils can generate lattices of DNA filaments interspersed with enzymes that immobilize and kill bacteria. However, inappropriately released NETs might be harmful owing to their cytotoxic, pro-inflammatory, and prothrombotic activity.

Tobias Fuchs and colleagues identified two DNases (DNase 1 and DNase- $\gamma$, also known as DNase 1-like 3) that independently degrade NETs. DNase 1 is expressed by nonhaematopoietic tissues and preferentially cleaves protein-free DNA, whereas DNase 1-like 3 is secreted by immune cells and targets DNA-protein complexes, such as nucleosomes.

In wild-type mice, NET formation associated with chronic neutrophilia was well tolerated. Similarly, mice with a single deficiency of either DNase (Dnase ${ }^{{ }^{-/}}$ or Dnase $13^{---}$) showed no signs of distress. However, double-knockout mice died within 6 days of induction of neutrophilia, indicating that either DNase is required to prevent adverse outcomes.

The double-knockout mice with chronic neutrophilia had intravascular NET clots with entrapped erythrocytes that fully or partially occluded blood vessels in the lungs, liver, and kidneys. Large aggregates of NETs have been reported in synovial fluid and pancreatic ducts of patients with gouty arthritis or pancreatitis, respectively. In the Science article, Fuchs and co-workers detected NET clots in blood vessels of two patients with sepsis.

"DNA strands from NETs can form clots that are sufficient to occlude blood vessels," concludes Fuchs. Of note, "the formation of NET clots occurs in the absence of platelets". This observation is important because NETs were previously thought to be a stimulus for classical thrombus formation involving activation of the coagulation cascade and platelets.

In conditions such as chronic neutrophilia or septicaemia, intravascular NETs can form clots that cause damage to erythrocytes and organs. Acquired or genetic defects in DNases, the body's natural protection mechanism, can delay the degradation of NETs and thereby precipitate disease.

"Clots of NETs are not inhibited by antiplatelet and anticoagulant therapies, but are digested by plasma DNases," observes Fuchs. Therefore, "DNases may provide an alternative antithrombotic therapy for patients in the future". 